\title{
Fascial system
}

Bruno Bordoni, Fabiola Marelli, Bruno Morabito, Roberto Castagna, Beatrice Sacconi, Paul Mazzucco

\section{Source}

Bruno Bordoni, Fabiola Marelli, Bruno Morabito, Roberto Castagna, Beatrice Sacconi, Paul Mazzucco. (2018). New Proposal to Define the Fascial System. Complement Med Res, vol. 25 (4), 257-262. doi:10.1159/000486238.

The fascia is any tissue that contains features capable of responding to mechanical stimuli. The fascial continuum is the result of the evolution of the perfect synergy among different tissues, capable of supporting, dividing, penetrating and connecting all the districts of the body, from the epidermis to the bone, involving all the functions and organic structures. The continuum constantly transmits and receives mechanometabolic information that can influence the shape and function of the entire body. These afferent/efferent impulses come from the fascia and the tissues that are not considered as part of the fascia in a biunivocal mode. 